\title{
Radiation driven winds from CV accretion disks
}

\author{
Daniel Proga, Janet E. Drew \\ Imperial College of Science, Technology and Medicine, Blackett \\ Laboratory, Prince Consort Road, London SW7 2BZ, UK \\ James M. Stone \\ Department of Astronomy, University of Maryland, College Park \\ $M D$ 20742, USA
}

\begin{abstract}
We present some initial results of our numerical, 2D hydrodynamical models of line driven flows from the accretion disk in cataclysmic variables. We assume the disk radiation pressure pushes out the isothermal material from a flat, geometrically thin, Keplerian disk.

We calculate the disk radiation field using the surface brightness of a standard " $\alpha$ disk" (Shakura \& Sunyaev 1973). We do not include a bright boundary layer in the calculations. We approximate the total radiative line acceleration, adopting the formalism due to Castor, Abbott, \& Klein (1975). We use our generalized 2D version of their force multiplier. The multiplier is still described by two parameters representing the number of lines and the ratio of optically thin to optically thick lines. The main modification of the original CAK force multiplier is in the depth parameter, which is now a function of the gradients of two velocity components instead of the single velocity gradient as in the $1 \mathrm{D}$ case.

We investigate how the disk structure and mass loss rate depend on the disk and central star luminosity, and boundary conditions such as the disk density.

We find that transonic flows from disks do not relax toward steady states. However, their time averaged properties become constant after some time. Our models show that most of mass loss originates from close to the central star - a few stellar radii. Models without a central star radiation field produce flows more vertical than models in which one is present. However, other global, time averaged properties of flows such as the total wind mass, the wind mass loss rate, and velocity are similar. The ratio between the wind mass loss and disk accretion rate increase rapidly with the accrection rate.
\end{abstract}

\section{References}

Castor, J.I., Abbott, D.C., \& Klein, R.I. 1975, ApJ, 195, 157

Shakura, N.I., \& Sunyaev, R.A. 1973, A\&A, 24, 337 